\title{
Publisher's Note: Very low velocity ion slowing down in binary ionic mixtures: Charge- and mass-asymmetry effects [Phys. Rev. ST Accel. Beams 13, 101302 (2010)]
}

Patrice Fromy, Bekbolat Tashev, and Claude Deutsch
(Received 13 October 2010; published 19 October 2010)

DOI: 10.1103/PhysRevSTAB.13.109901 PACS numbers: 34.50.Bw, 52.27.Cm, 52.40. - w, 52.20.- j, 99.10.Fg

This paper was published online on 1 October 2010 with an omission of an Acknowledgment. The following Acknowledgment has been added to the online article as of 15 October 2010: "This work has been performed under the auspices of CEA/EURATOM Contract No. V35599.001 for which the authors are deeply grateful."” 